infections. Recent advances in this technology and the computational tools developed in their wake provide unique opportunities to build reference cell atlases that can be used to interpret new single-cell RNA-sequencing (scRNA-seq) data and systematically compare data sets derived from different models or therapeutic conditions.

Materials and Methods We have developed ProjecTILs (https:// github.com/carmonalab/ProjecTILs), a novel computational method to project new data sets into a reference map of $\mathrm{T}$ cells, enabling their direct comparison in a stable, annotated system of coordinates. ProjecTILs enables the classification of query cells into curated, discrete states, but also over a continuous space of intermediate states. We illustrate the projection of several data sets from recent publications over two crossstudy murine $\mathrm{T}$ cell reference atlases: the first describing tumor-infiltrating $\mathrm{T}$ lymphocytes (TILs), the second characterizing acute and chronic viral infection.

Results ProjecTILs accurately predicted the effects of multiple perturbations, including the ablation of genes controlling T cell differentiation, such as Tox, Ptpn2, miR-155 and Regnase-1, and identified novel gene programs that were altered in these cells (such as a Lag3-Klrc1 inhibitory module), revealing mechanisms of action behind these immunotherapeutic targets and opening new opportunities for the identification of novel targets. By comparing multiple samples over the same reference map, and across alternative embeddings, our method allows exploring the effect of cellular perturbations (e.g. as the result of therapy or genetic engineering) in terms of transcriptional states and altered genetic programs.

Conclusions The proposed computational method will likely contribute to reveal the mechanisms of action of experimental immunotherapies and guide novel therapeutic interventions in cancer and beyond.

Disclosure Information M. Andreatta: None. S.J. Carmona: None.

\section{P03.22 REPOLARIZATION OF TUMOR-ASSOCIATED MACROPHAGES FOR IMMUNOTHERAPY OF TUMORS WITH DIVERSE MAJOR HISTOCOMPATIBILITY COMPLEX CLASS I EXPRESSION}

A Piataková*, I Poláková, M Šmahel. Department of Genetics and Microbiology, Faculty of Science, Charles University, BIOCEV, Prague, Czech Republic

\subsection{6/jitc-2020-ITOC7.60}

Background Depletion of tumor-associated macrophages (TAMs), which are regarded as M2, pro-tumor cells, is one of the strategies for cancer treatment. However, repolarization of TAMs to the M1 anti-tumor phenotype could constitute an immunotherapeutic alternative for tumors with defective major histocompatibility complex class I (MHC-I), where the anti-tumor effect of cytotoxic $\mathrm{CD}^{+} \mathrm{T}$ cells could be limited.

Materials and Methods In this study, we characterized TAMs from mouse tumor models of human papillomavirus 16-associated tumors, characterized by either reversibly (TC-1/A9) or irreversibly (TC-1/dB2m) downregulated MHC-I expression. Tumors were treated with DNA immunization against the papillomaviral E7 oncoprotein combined with intraperitoneal injection of the synthetic oligodeoxynucleotide ODN1826, a Toll-like receptor 9 agonist. TAMs were characterized ex vivo by flow cytometry. In vitro, $\mathrm{F} 4 / 80^{+}$TAMs from naïve tumors were stimulated to $\mathrm{M} 1$ or $\mathrm{M} 2$ phenotype and co-cultures with TC-1/A9 or TC-1/dB2m cells were established. The cytotoxic effect of polarized TAMs was investigated, and the role of nitric oxide (NO) and tumor necrosis factor (TNF)- $\alpha$ was examined. Finally, interleukin (IL)-10, IL-12 and TNF- $\alpha$ concentrations were determined by ELISA in the culture media from polarized TAMs.

Results We demonstrated that TAMs infiltrated both tumor types and this effect was moderately enhanced after combined immunotherapy. Increase in MHC-II molecules, broadly regarded as an M1 marker, was observed solely in TAMs from treated TC-1/A9 tumors. In contrast, TAMs from TC-1/ $\mathrm{dB} 2 \mathrm{~m}$ tumors expressed high MHC-II levels, regardless of the treatment. Therefore, the new $\mathrm{CD}_{3} 8^{+} / \mathrm{Egr}^{+}{ }^{+}$classification $^{1}$ was applied and showed to be a better descriptive parameter for M1/M2 TAMs, respectively, because the number of $\mathrm{Egr}^{+}$ TAMs decreased in both tumor types after combined immunotherapy. While CD $38^{+}$TAMs were significantly increased after treatment of TC-1/A9 tumors, they did not increase substantially in $\mathrm{TC}-1 / \mathrm{dB} 2 \mathrm{~m}$ tumors. In vitro, co-cultures with tumor cells resulted in increase of $\mathrm{NO}$ production by M1 TAMs. However, NO and TNF- $\alpha$ contributed to the cytotoxic effect only in TAMs from TC-1/A9 tumor. Finally, in vitro polarized M1 TAMs were able to produce TNF- $\alpha$ and IL-10 but not IL-12.

Conclusions Our results showed different effects of immunostimulation on cytotoxicity of TAMs from tumors with distinct MHC-I expression. While TAMs from TC-1/A9 tumors acquired M1 phenotype and became cytotoxic, TAMs from TC- $1 / \mathrm{dB} 2 \mathrm{~m}$ tumors were more resistant to repolarization. This project was supported by grants GA19-00816S provided by the Czech Science Foundation and LQ1604 provided by the Ministry of Education, Youth and Sports of the Czech Republic.

\section{REFERENCE}

1. Jablonski KA, Amici SA, Webb LM, Ruiz-Rosado JdD, Popovich PG, Partida-Sanchez S, Arellano M. Novel Markers to Delineate Murine M1 and M2 Macrophages. PLOS ONE 2015; 10(12); 1-25.

Disclosure Information A. Piataková: None. I. Poláková: None. M. Šmahel: None.

\section{P03.23 EVOLUTION OF THE IMMUNE LANDSCAPE WITHIN PARTIALLY CONTROLLED MURINE MELANOMA}

C Qing*, E Ghorani, I Solomon, F Gálvez-Cancino, F Vargas, K Peggs, S Quezada. Cancer Institute, University College London, London, UK

\subsection{6/jitc-2020-ITOC7.61}

Background Regulatory $\mathrm{T}$ cell (Treg) depletion with antibodies against CD25 is effective in tumor models but response rates are low in poorly infiltrated B16 melanomas. Combination with a tumor vaccine enhances efficacy, but relapse usually occurs following partial control, similar to what is seen clinically. How resistance develops is unknown.

Materials and Methods C57BL/6 mice were injected subcutaneously with B16 cells. Treatments included a depleting mouse IgG2a $\alpha \mathrm{CD} 25$ antibody and/or a genetically modified, granulocyte-macrophage colony-stimulating factor (GM-CSF) secreting whole B16 tumor vaccine (Gvax). Changes in the immune landscape were assessed with high dimensional flow cytometry. 